\title{
Bi-directional Message Mapping for DER and DMS Interoperability
}

\author{
Jian $\mathrm{Su}^{1, \mathrm{a}^{*}}$, Haitao $\mathrm{Liu}^{1, \mathrm{~b}}$ and Xueyuan $\mathrm{Su}^{2, \mathrm{c}}$ \\ ${ }^{1}$ China Electric Power Research Institute, Beijing 100192,China \\ ${ }^{2}$ Xiamen Electric Power Bureau, Xiamen 361004, China \\ a sujian@epri.sgcc.com.cn, ${ }^{\text {b } I h t c n @ e p r i . s g c c . c o m . c n ~}$
}

Keywords: Message Mapping, CIM, Distributed Energy Resources, DMS.

Abstract. The Common Information Model (CIM) is an abstract data model that use in Distribution Management System (DMS) applications. And DER devices use IEC61850 information model. Because DMS and DER systems use different data modal, the bi-directional message mapping method need to study. The article analyzes the scenario of DER operation and management for DMS applications, then established bi-directional message mapping method for DER and DMS interoperability.

\section{Introduction}

In recent years, distributed energy resources (DERs) have been rapid development. The capacity of DERs are small, usually tens of kilowatts to tens of megawatts, so it commonly connects to distribution network directly [1]. The manufacturers of DER devices are facing the age-old issues of what communication standards and protocols to provide to their customers for monitoring and controlling DER devices, in particular when they are interconnected with the electric utility system. To solve this problem, the IEC61850-7-420 standard was proposed to information modeling for DER[2]. IEC61850-7-420 includes a lot of logical nodes that are intended for use with DER.

The distribution management system (DMS) applications use Common Information Model (CIM) as information model [3]. The CIM, as defined by the IEC 61970, IEC 61968 and IEC62325 series of standards, is currently well suited for intra-operability of applications within a DMS. The CIM is an abstract data model that can be used to represent the major objects in an electric utility enterprise. The CIM began as a common model to enable intra-operability of Energy Management System (EMS) functions and to facilitate the exchange of EMS data models among utilities. Later, the CIM was extended to model the distribution system objects and to define system interfaces to assist with inter-operability of DMS applications. Though the objects for the distribution system are well defined, they do not cover DER systems.

The DMS needs to manage the DERs but DMS and DER systems use different data modal. So when the DMS and DER management systems to interoperate, the mapping between different information models need to study.

The article analyzes the scenario of DER operation and management for DMS applications, then established bi-directional message mapping method for DER and DMS interoperability.

\section{Distribution network information interaction system architecture}

Commonly the DMS and Distribution Automation provide for Volt/Var management, Distributed Power Flow Calculation/Distributed State Estimation, Fault Location, Isolation and Service Restoration and others. For DERs management, DMS also needs Distributed Energy Resource Management (DERM) functions that provide capabilities to optimally manage and control distributed energy resources to meet various economics and reliability objectives. DERM can be a standalone system or a module of DMS. DMS with DER management system architecture is as Fig.1. 


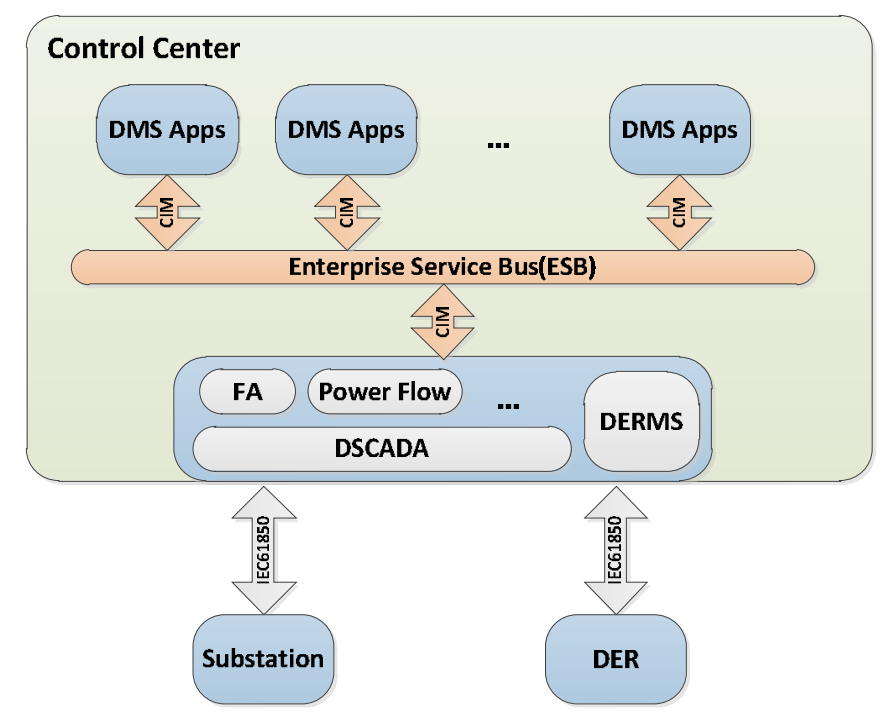

Fig.1 DMS with DER Management System Architecture

DERM system communicates with DER devices in the field by SCADA that uses IEC61850. Because the DMS applications use CIM to interact, DERM system need convert IEC61850 data into CIM data.

\section{CIM extension for DER}

Individual DER device's capacity is generally relatively small, so they commonly need the multi-group, multi-level interconnection, and then connect to utility power system via a common connection point (PCC) [4]. If the DMS master station manages each DER device directly, with the increasing number of DER devices, the amount of communication data will increase rapidly. The DMS master station and communication network will bear the enormous load. Each DER device has little effect on the overall system operation, but the multi-group, multi-level interconnection DER system has a greater impact. From the view of DMS advanced applications, they care about the overall characteristics of the DER system. So the multi-group, multi-level interconnection DER system is modeled as a control object is the most reasonable and most effective method.

\section{Bi-directional message mapping method}

IEC61850 models and CIM have different semantics, though they are designed by using object-oriented method. A message in IEC 61970 is coded according to Resource Description Framework (RDF) Schema and a message in IEC 61850 is coded according to XML Schema. DMS needs the message data exchange, that involves conversion of data based on IEC 61970 to IEC 61850, and vice versa. The data exchange commonly applies mapping rules.

Pre-defined mapping rules set can be getting by analyzing the business entities and semantic constraints for a specific business application, and then by adding user-defined set of mapping rules, can form a complete set of message mapping rules to meet specific business applications. Mapping rules include not only the CIM and IEC 61850 model's element corresponding to each other (class, logical nodes, attributes, etc.), but also the correspondence of relationships, such as many-to-one, one-to-many, one-to-one etc. Using the mapping rule set to convert the CIM, IEC61850 model message is shown as Fig. 2. 


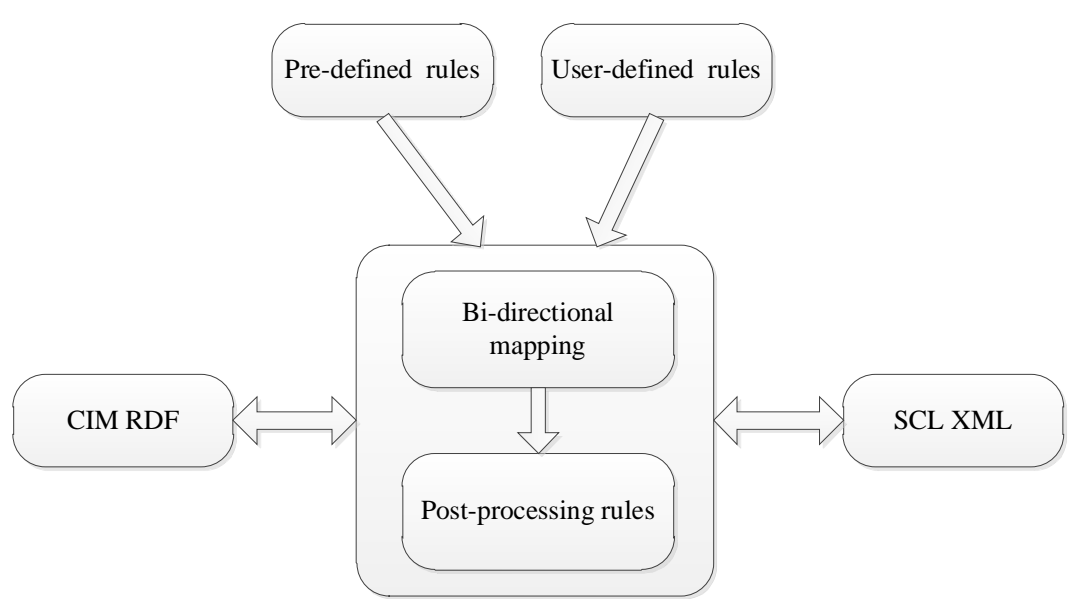

Fig. 2 Bidirectional mapping of CIM and IEC61850 model message

Extended CIM model containing DER can do bidirectional mapping of message with IEC61850. The steps are as follow.

(1)Depending on the specific version of CIM and IEC61850 models, identify and determine the mapping relationship between model elements, accessing to a predefined set of mapping rules through the analysis of the business entities and semantic constraints.

(2)For a specific business application, user-defined mapping rules can be added. User achieves this mapping rules by the using the corresponding program.

(3)Using the mapping rules to complete CIM RDF and SCL XML based messages bidirectional mapping process. First mapping identified element by mapping rule; for the result after the conversion, and then to supplement some of the properties that the required standards of converting the results by mapping rules and so on.

\section{Data-level mapping}

(1) Mapping between IEC61850 CDC and CIM data class

IEC61850-7-2 defines the basic data type used. For example, IEC61850 defines INT8, INT16, INT24, INT32, INT128, INT8U, INT16U, INT24U, INT32U total of 9 different range of integers, while mapped to CIM is not required so much integer type. Typically they map to int (a signed integer, usually 4-byte integer) and uint (unsigned integer).

IEC61850 common data class (CDC) is composed of structured data based on basic data types, generally using the appropriate basic data characterizing the main information, and then add some quality, time attribute and other ancillary properties. The ancillary properties is to ensure the correctness of communication, so when mapping only need to map the common data type of the main information. In INS (integer status, INT32), for example, can be directly mapped to CIM property stVal int type, and don't need to mapping q (Quility), t (TimeStamp) and other attributes.

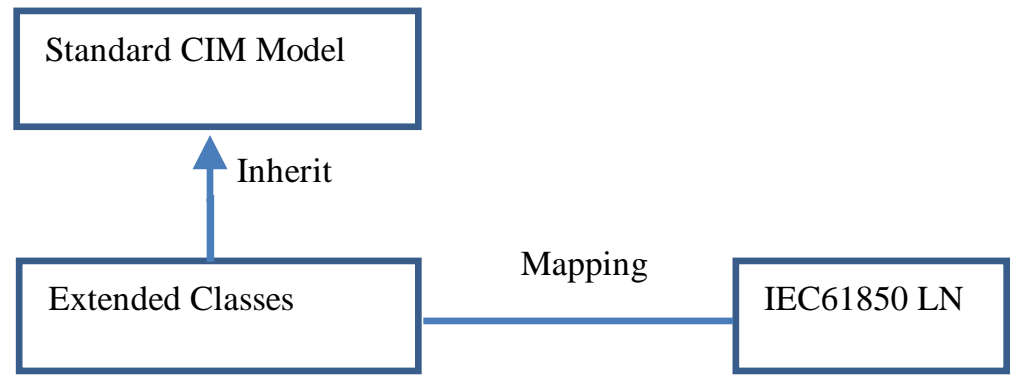

Fig. 3 The mapping between the IEC61850 LN and CIM model

(2) Mapping between IEC61850 LN and CIM object class 
The mapping is through differentiated analysis, the function of each logical node for re-abstraction and division, and with the original class CIM model for comparison, expands the corresponding class and builds relationships, to express mapping, as Fig. 3. Due to the different emphases CIM and IEC61850 information modeling when, IEC61850 logic may be one to one mapping node with CIM class map, or it may be many-to-many mappings.

(3) Mapping attributes between IEC61850 LN and CIM

The map is mainly based on semantic consistency to be mapped. DER extended CIM model extended as far as possible the use of class attributes consistent with the IEC61850 name of the property to characterize consistent semantics. Different names and semantics are not completely identical to the corresponding attribute mapping table by correspondence.

\section{Conclusion}

By analysis the scenario of the message interaction between DMS and DER, a bidirectional messaging mapping method based on data exchange was proposed to get through the information exchange barrier between IEC61850 standard and IEC61970/ 61968 standard. But the data-level mapping need to deep research that includes the relationship between data types, data objects, as well as IEC61850 data merge to CIM data, CIM data decompose to IEC61850 data.

\section{Acknowledgement}

In this paper, the research was sponsored by the State Grid Corporation of China (Project No. PDB 52134014009A).

\section{References}

[1] S. Chowdhury, S. P. Chowdhury, P. Crossley, Microgrids and Active Distribution Networks, The Institution of Engineering and Technology, London, UK, 2009.

[2] IEEE, IEC 61850 Communication networks and systems for power utility automation,Ed.2.

[3] IEEE, IEC 61970 Energy management system application program interface (EMS-API), Ed.1.

[4] IEEE, Standard 1547 Interconnecting Distributed Resources with Electric Power Systems, (2009).

[5] Ming Ding,Zhengkai Zhang,Xuefeng Guo, CIM Extension of Microgrid Energy Management System,APPEEC Power and Energy Engineering Conference, 2009,pp:1-6

[6] Latisko Goran, Bhati Devi, Landenberger Volker, Application of IEC61970 and IEC61968 at KCP\&L Smart Grid Demonstration Project, IEEE PES Innovative Smart Grid Technologies Conference (ISGT),2014,pp:1-5

[7] D. Becker, T.L. Saxton, M. Goodrich, CIM standard for dynamic model exchange, Power and Energy Society General Meeting, 2010,IEEE,pp:1-3

[8] E. Lambert , A. Quéric, Use of CIM for EDF Distribution Automation, Power and Energy Society General Meeting, 2010 IEEE,pp:1-4

[9] T.D. Nielsen, S.A. Neumann, T.L. King, A methodology for managing model extensions when using the common information model for systems integration, Power Energy Society General Meeting,2009,IEEE,pp:1-5

[10] T.L. King, M.F. McGranaghan, Using and extending the CIM standards for advanced distribution applications,IEEE Power and Energy Society General Meeting - Conversion and Delivery of Electrical Energy in the 21st Century, 2008, pp:1-3 